\title{
Periodic-orbit bifurcations as the origin of nuclear deformations
}

\author{
Ken-ichiro Arita \\ Department of Physics, Nagoya Institute of Technology, Nagoya 466-8555, Japan
}

(Dated: July 15, 2005)

\begin{abstract}
Semiclassical analysis of shell structures in realistic nuclear potentials are presented using periodicorbit theory. We adopted $r^{\alpha}$ potential model and examined classical-quantum correspondence using Fourier transformation technique. Spin-orbit coupling is also taken into account in the model Hamiltonian. Gross shell structure for a certain combination of surface diffuseness and spin-orbit parameters are investigated and its relation to pseudospin symmetry is discussed. Analysis of superdeformed shell structure in realistic model is also presented.
\end{abstract}

PACS numbers: 21.60.-n, 03.65.Sq, 31.15.Gy

\section{SHELL STRUCTURE AND PERIODIC ORBITS}

Nuclear deformations are intimately related with shell structures in single-particle energy spectra of deformed Hamiltonian. Using the semiclassical theory, quantum level density $g(E)$ can be represented in terms of classical periodic orbits, and one obtains the trace formula $[1,2]$

$$
g(E) \sim g_{0}(E)+\sum_{\beta} A_{\beta}(E) \cos \left[\frac{S_{\beta}(E)}{\hbar}-\nu_{\beta}\right] .
$$

$g_{0}(E)$ is average part of the level density, and the oscillating part is expressed as the sum over all periodic orbits $\beta$ in corresponding classical Hamiltonian system. $S_{\beta}=\oint_{\beta} \boldsymbol{p} \cdot d \boldsymbol{r}$ is action integral along the orbit $\beta$, and $\nu_{\beta}$ is Maslov phase determined by the number of conjugate points along the orbit.

In the trace formula, each periodic orbit contribution is an oscillatory function of energy since action $S_{\beta}(E)$ is an increasing function of $E$. The energy scale of this oscillation is given by $\delta E \approx 2 \pi \hbar / T_{\beta}$. From this relation, one can see that short periodic orbits (having small $T_{\beta}$ ) is associated with gross structure (having large $\delta E$ ).

The amplitude factor $A_{\beta}$ in the trace formula (1) has significant dependence on the stability of the orbit. In standard stationary-phase approximation, it is proportional to the stability factor;

$$
A_{\beta} \propto \frac{1}{\sqrt{\left|\operatorname{det}\left(M_{\beta}-I\right)\right|}}
$$

where $M_{\beta}$ represents symmetry-reduced monodromy matrix[3], which characterizes linear stability of the periodic orbit. Varying external parameters in the Hamiltonian, each periodic orbit continuously changes its properties, and one of the eigenvalues of $M_{\beta}$ might eventually approaches 1 . At this point, a continuous family of quasi-periodic orbits appear in neighborhood of the periodic orbit $\beta$ in direction to eigenvector belonging to the above unit eigenvalue of $M_{\beta}$. It usually accompany the appearance of new periodic orbit from that local family (or, inversely, disappearance of another periodic orbit into the family), namely bifurcation of periodic orbit occur at this point. One should also note that this periodic-orbit bifurcation is related with the restoration of local dynamical symmetry in neighborhood of the periodic orbit. Since these quasi-periodic orbits make coherent contribution in the periodic orbit sum, we can expect a significant enhancement of shell effect in quantum level density. Actually, our previous works $[4,5]$ clearly show that the bifurcations of short periodic orbits play essential role in emergence of gross shell structures. The divergence of stability factor (2) at bifurcation point is due to the breakdown of standard stationary-phase method, and can be remedied by using suitable approximation for trace integral such as uniform approximations [6, 7] and improved stationary phase method $[8]$.

\section{THE $r^{\alpha}$ POTENTIAL MODEL}

For nuclei and metallic clusters, central part of the mean field is well described by Woods-Saxon potential. For stable nuclei, Woods-Saxon potential can be further approximated by much simpler potential with $r^{\alpha}$ radial dependence[9]

$$
V_{\mathrm{WS}}=-\frac{W}{1+\exp ((r-R) / a)} \approx-W+U \cdot(r / R)^{\alpha} .
$$

Here, the parameter $\alpha$ controls the surface diffuseness. With typical value of Woods-Saxon parameters for nuclei, we found that the quantum energy spectra of $r^{\alpha}$ model nicely reproduce those of Woods-Saxon model up to about $-10 \mathrm{MeV}$.

Shifting the zero-point energy, we write the $r^{\alpha}$ model Hamiltonian as

$$
H=\frac{p^{2}}{2 M}+U \cdot\left(\frac{r}{R f(\theta, \phi)}\right)^{\alpha} .
$$

The advantage of the $r^{\alpha}$ model is its scaling property. Since equations of motion (EOM) are invariant under scale transformation

$$
\boldsymbol{r} \rightarrow c^{\frac{1}{\alpha}} \boldsymbol{r}, \quad \boldsymbol{p} \rightarrow c^{\frac{1}{2}} \boldsymbol{p}, \quad t \rightarrow c^{\frac{1}{\alpha}-\frac{1}{2}} t, \quad(\text { as } E \rightarrow c E)
$$

one has the same set of periodic orbits in all energy surfaces. The action integral has simple energy dependence

$$
S_{\beta}(E)=\oint_{\beta} \boldsymbol{p} \cdot d \boldsymbol{r} \propto \sqrt{M E} \cdot R(E / U)^{1 / \alpha}=\hbar \mathcal{E},
$$


where we define scaled energy $\mathcal{E}$ as

$$
\mathcal{E}=\frac{\sqrt{M U R^{2}}}{\hbar}\left(\frac{E}{U}\right)^{1 / \alpha+1 / 2} .
$$

We also define scaled period by $\tau_{\beta}=S_{\beta}(E) / \hbar \mathcal{E}$, which is a scale-invariant parameter proper to the periodic orbit $\beta$. Then we obtain semiclassical level density with scaled energy

$$
g(\mathcal{E})=g(E) \frac{d E}{d \mathcal{E}}=g_{0}(\mathcal{E})+\sum_{\beta} A_{\beta}(\mathcal{E}) \cos \left(\mathcal{E} \tau_{\beta}-\nu_{\beta}\right)
$$

This simple form is convenient for Fourier analysis. The Fourier transform of the trace formula

$$
F^{\mathrm{cl}}(\tau)=\int d \mathcal{E} e^{i \tau \mathcal{E}} g(\mathcal{E})=F_{0}(\tau)+\pi \sum_{\beta} e^{i \nu_{\beta}} \hat{A}_{\beta} \delta\left(\tau-\tau_{\beta}\right)
$$

is a function having peaks at scaled period $\tau_{\beta}$ of classical periodic orbits. It suggests that we can extract information on classical periodic orbits from the Fourier transform of quantum level density

$$
F^{\mathrm{qm}}(\tau)=\int d \mathcal{E} e^{i \tau \mathcal{E}} g(\mathcal{E})=\sum_{i} e^{i \tau \mathcal{E}_{i}},
$$

where $\mathcal{E}_{i}$ is related with quantum energy level $E_{i}$ via Eq. (7).

For description of nuclear shell structure, it is very important to incorporate strong spin-orbit coupling in the mean field. One can also apply periodic orbit theory to a system with spin-orbit coupling, if we utilize $\mathrm{SU}(2)$ coherent state path integral method[10-12]. It introduces classical variable corresponding to a spin degree of freedom. Let us write spin vector in polar coordinate as $s=s(\sin \vartheta \cos \varphi, \sin \vartheta \sin \varphi, \cos \vartheta)$, with $s=\hbar / 2$. One can choose spin canonical variable as $\left(q_{s}, p_{s}\right)=(\varphi, s \cos \vartheta)$ and obtains the Poisson bracket relations $\left\{s_{i}, s_{j}\right\}_{\text {P.B. }}=\epsilon_{i j k} s_{k}$, which exactly correspond to the commutation relation of quantum spin operators. The Hamiltonian with spin-orbit coupling is written as

$$
H=\frac{p^{2}}{2 M}+U\left(\frac{r}{R f(\theta)}\right)^{\alpha}-\frac{2 \kappa R^{2}}{\hbar^{2}}\left(\nabla V_{s o}(\boldsymbol{r}) \times \boldsymbol{p}\right) \cdot \boldsymbol{s} .
$$

In order to make use of scaling property of the central potential, we adopt spin-orbit potential

$$
V_{\mathrm{so}}(\boldsymbol{r})=U\left(\frac{r}{R f(\theta)}\right)^{\alpha^{\prime}}
$$

and choose the diffuseness parameter $\alpha^{\prime}=\alpha / 2+1$. With this choice of spin-orbit coupling, the classical EOM are invariant under transformation (5) for some special classes of periodic orbits whose spin does not change its orientation; $\dot{\boldsymbol{s}}=0$. For axially symmetric or reflectionsymmetric shapes, such orbits exist as exact solutions of the EOM. Using the Hamiltonian (11) with suitable $\alpha$ and $\kappa$, we can reproduce realistic shell structures both for spherical and deformed nuclei.

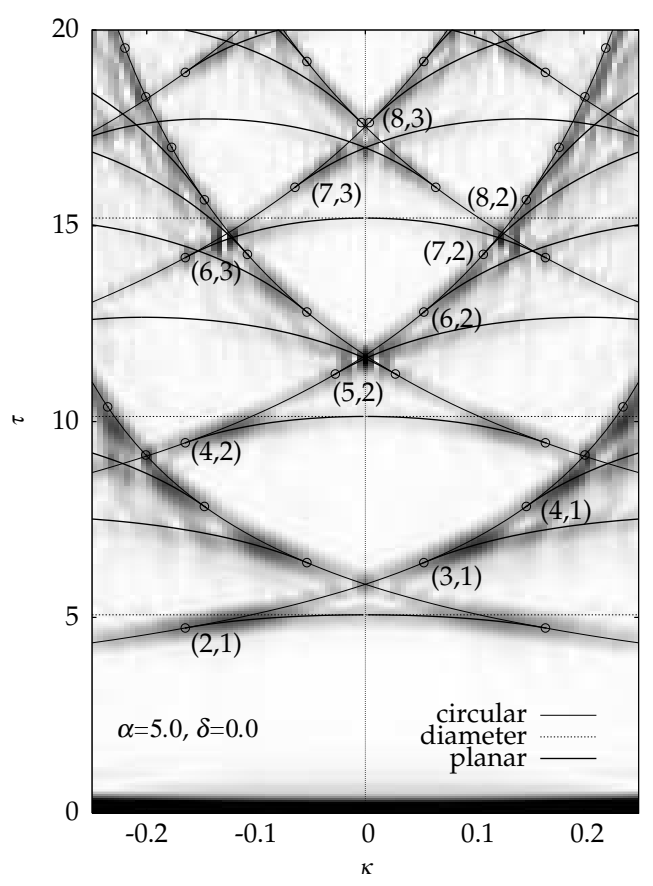

FIG. 1: Scaled periods $\tau_{\beta}$ of classical periodic orbits plotted as functions of spin-orbit parameter $\kappa$. Open circles represent the bifurcation points. The background gray-scale image show the Fourier transform of quantum scaled-energy level density; the Fourier amplitude $|F(\tau ; \kappa)|$ have peaks in dark regions.

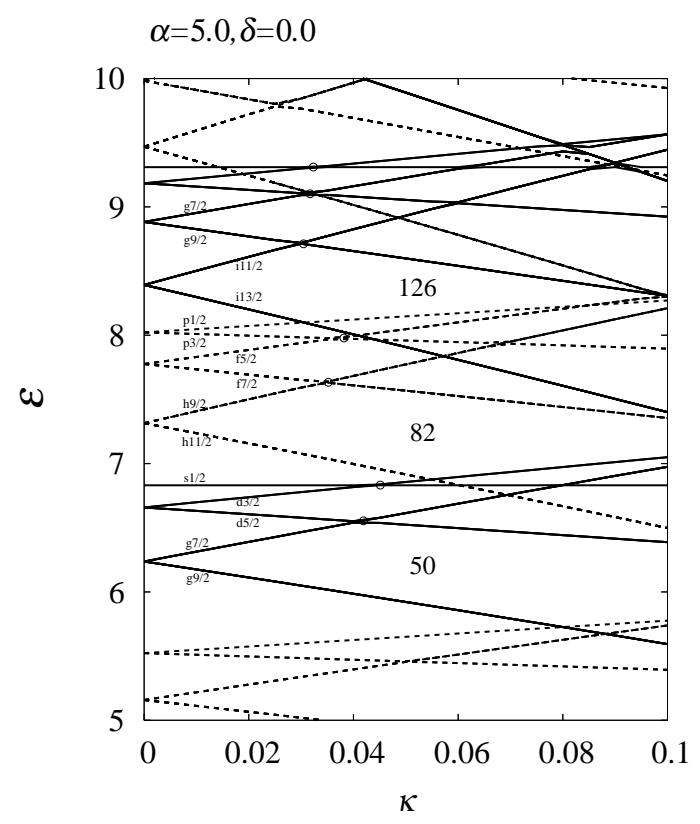

FIG. 2: Single-particle level diagram against spin-orbit parameter $\kappa$. Solid and broken curves represent even and odd parity levels, respectively. Open circles indicate the crossing points of pseudo spin-orbit partners. 

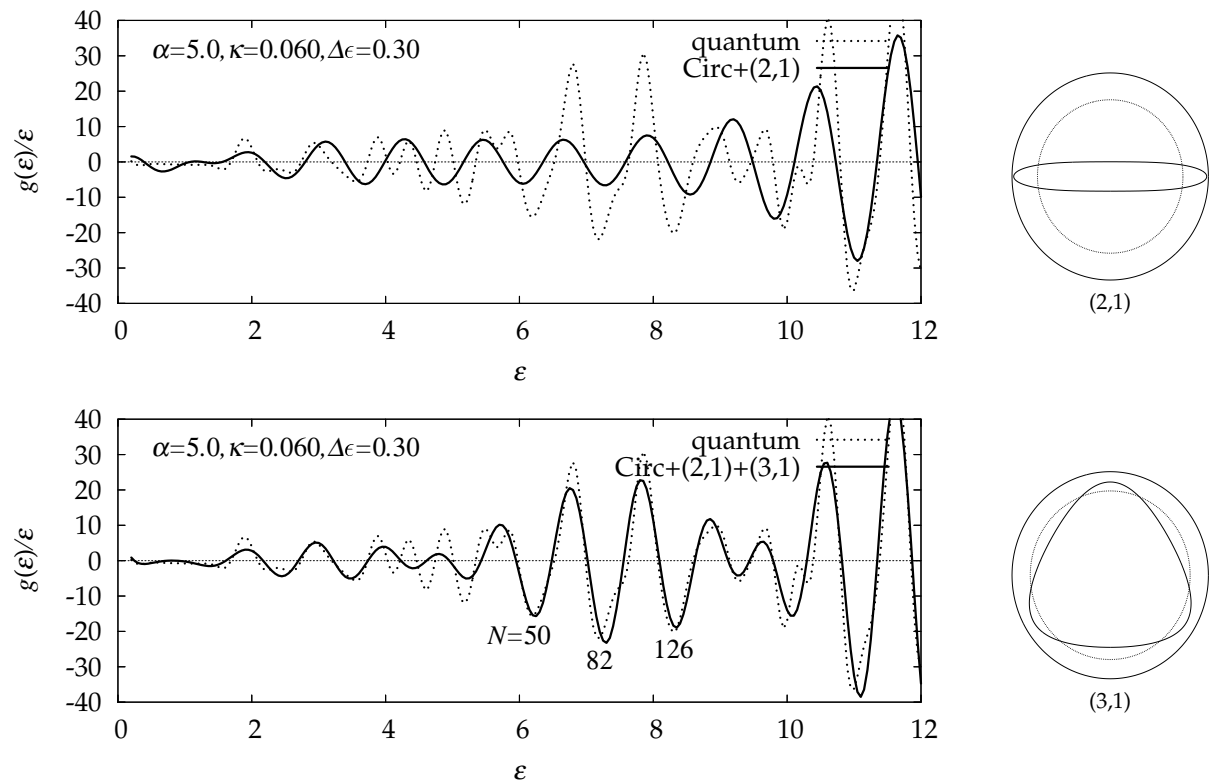

FIG. 3: Oscillating part of coarse-grained quantum level density is compared with semiclassical trace formula. In the top panel, the shortest circular orbit and oval orbit $(2,1)$ are considered in the periodic orbit sum. In the bottom panel, longer circular orbit and triangular orbit $(3,1)$ are also taken into account. (In this plot, amplitude $A_{\beta}$ and Maslov phase $\nu_{\beta}$ in trace formula (8) are determined so that they best fit the quantum result.) The right panels display the classical periodic orbits, where the outermost circle represents the boundary of classically accessible region.

\section{NUMERICAL RESULTS}

In Fig. 1, we compare scaled period $\tau_{\beta}$ 's of classical periodic orbits with Fourier transform of quantum level density. Absolute value of Fourier transform $|F(\tau)|$ are displayed by gray-level image and it exhibits peaks at dark regions. We found that all important peaks of Fourier amplitude are located at scaled periods $\tau_{\beta}$ of classical periodic orbits, displayed by curves, and they are significantly enhanced around the bifurcation points. Especially, the Fourier amplitude corresponding to circular and $(3,1)$ orbits are strongly enhanced around the bifurcation point $\kappa \simeq 0.05$. It implies a significant growth of gross shell structure around $\kappa \approx 0.05$.

Figure 2 shows the single-particle level diagram against spin-orbit parameter $\kappa$. As we expected from the Fourier analysis, remarkable shell structure emerge at about $\kappa \simeq$ 0.05 , with large energy gaps corresponding to well known nuclear magic numbers 50, 82 and 126. One should also note that pseudo spin-orbit partners degenerate at almost same values of $\kappa$. These degeneracies are responsible for the occurrence of the above mentioned large energy gaps. This implies that pseudospin symmetry[13] is approximately restored in this region. As we stated in $\S 1$, local restoration of dynamical symmetry is usually accompanied by bifurcations of classical periodic orbits. Therefore, we can expect that the pseudospin symmetry might be connected with periodic orbit bifurcation.

Pseudospin symmetry arise for a certain combination of spin-orbit and orbit-orbit force parameters in Nilsson Hamiltonian. In the Nilsson model, orbit-orbit force is introduced to describe sharp surface of potential well and therefore connected with surface diffuseness. In $r^{\alpha}$ potential model, bifurcations of periodic orbits occur for certain combinations of diffuseness parameter $\alpha$ and spinorbit coupling parameter $\kappa$. Therefore, it should be quite natural to consider that bifurcation of $(3,1)$ orbit which occurs at $\kappa \simeq 0.05$ is a semiclassical origin of gross shell structure related with pseudospin symmetry.

In order to see the importance of $(3,1)$ orbit for gross shell structure, we analyze the coarse-grained quantum level density. In the left panels of Fig. 3, oscillating part of quantum level density coarse-grained with a smoothing width $\Delta \mathcal{E}=0.3$ is plotted with dotted curve. In the periodic orbit sum, contribution of long orbits are suppressed by coarse-graining. For $\Delta \mathcal{E}=0.3$, orbits with scaled periods $\tau_{\beta}>2 \pi / \Delta \mathcal{E} \simeq 20$ are sufficiently suppressed. The solid curve in the upper panel of Fig. 3 represents the semiclassical level density where only shortest circular orbit (whose spin is anti-parallel to the orbital angular momentum) and oval orbit $(2,1)$ (see the right upper part of Fig. 3) are taken into account. It apparently fail to reproduce the gross pattern of quantum result. Next we take into account the contribution of longer circular orbit (whose spin is parallel to the orbital angular momentum) and triangular orbit $(3,1)$ (see the right lower part of Fig. 3). Then we can nicely reproduce the gross structure of quantum level density as shown in the lower panel of Fig. 3. Thus we see that this bifurcating orbit actu- 


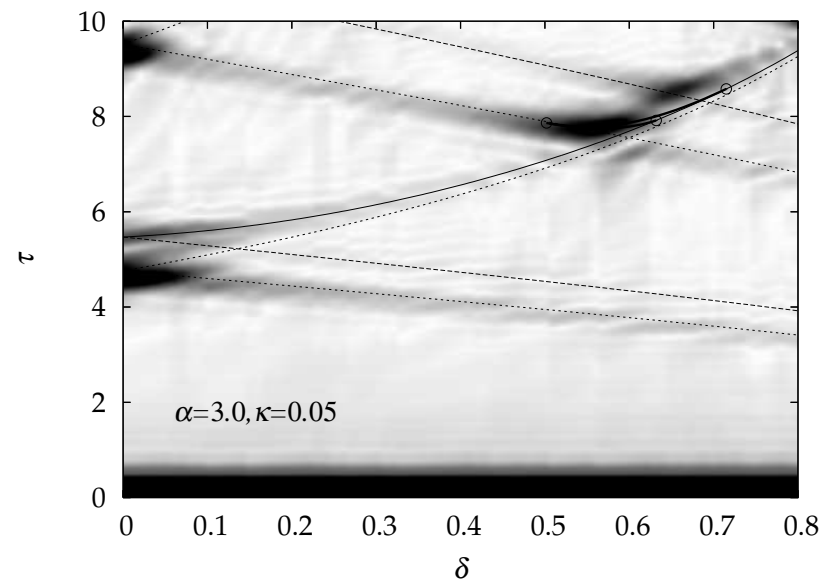

FIG. 4: Scaled period $\tau_{\beta}$ of classical periodic orbits plotted as functions of deformation parameter $\delta$. Open circles represent the bifurcation points. In the background, Fourier transform of level density $|F(\tau, \delta)|$ is plotted by gray-scale image. The Fourier amplitude exhibits peaks at dark regions.
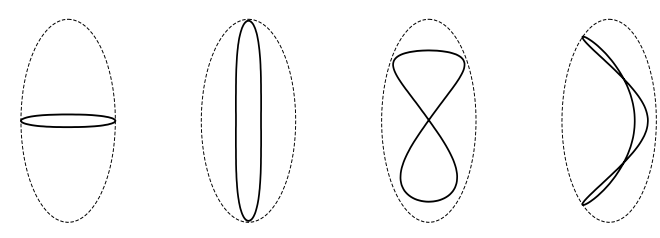

$(4,2,1) \mathrm{a}$

$(4,2,1) b$

FIG. 5: Periodic orbits responsible for superdeformed shell structure in $r^{\alpha}$ model with $\alpha=3.0$ and $\kappa=0.05$.

ally play essential role in characterizing the gross shell structure.

Using the $r^{\alpha}$ model with spin-orbit coupling, we also analyze the semiclassical origin of superdeformed shell structure. Figure 4 shows the Fourier transform of level density as function of deformation parameter $\delta=$ $3(\eta-1) /(2 \eta+1)$, connected with axis ratio $\eta=R_{z} / R_{\perp}$. We found significant enhancement of Fourier amplitude at $\delta=0.5 \sim 0.6$, corresponding to the bifurcations of periodic orbits shown in Figure 5. With increasing deformation, oval orbit lying around the short diameter occur period-doubling bifurcation and new orbits $(4,2,1) \mathrm{a}$ and $(4,2,1) \mathrm{b}$ emerge at $\delta \simeq 0.5$, and then they submerge into the oval orbit lying around the long diameter at $\delta>0.6$. This situation is quite similar to the case of the model without spin-orbit couplings[5].

\section{SUMMARY}

We applied periodic orbit theory to a realistic nuclear mean field potential model with spin-orbit coupling. The scaling property of $r^{\alpha}$ potential model enables us to investigate quantum-classical correspondence using Fourier transformation technique. We found nice quantum-classical correspondence, and significant roles of periodic-orbit bifurcations are clarified, also for our systems with spin-orbit coupling. Especially, $(3,1)$ orbit bifurcation which occur for a certain combination of diffuseness and spin-orbit parameters can be regarded as the semiclassical origin of gross shell structure related with pseudospin symmetry. Superdeformed shell structures are also examined and we found significant roles of bifurcations of periodic orbits which are similar to those in the model with no spin-orbit couplings. It implies that the semiclassical origin of superdeformed shell structure is essentially accounted for by models without spin-orbit couplings.
[1] M.C. Gutzwiller, J. Math. Phys. 8 (1967), 1979; 12 (1971), 343.

[2] M. Brack and R.K. Bhaduri, "Semiclassical Physics", (Addison-Wesley Reading, 1997).

[3] S.C. Creagh and R.G. Littlejohn, Phys. Rev. A44 (1991), 836.

[4] K. Arita and K. Matsuyanagi, Nucl. Phys. A592 (1995), 9.

[5] K. Arita, A. Sugita and K. Matsuyanagi, Prog. Theor. Phys. 100 (1998), 1223.

[6] A.M. Ozorio de Almeida and J.H. Hanney, J. of Phys. A20 (1987), 5873.
[7] M. Sieber, J. of Phys. A29 (1996), 4715.

[8] A.G. Magner et al, Prog. Theor. Phys. 102 (1999), 551; 108 (2002), 853.

[9] K. Arita, Int. J. of Mod. Phys. E13 (2004), 191.

[10] J. R. Klaudar, Phys. Rev. 19 (1979), 2349.

[11] T. Suzuki and H. Kuratsuji, J. Math. Phys. 21 (1980), 472.

[12] M. Pletyukhov and O. Zaitsev, J. Phys. A36 (2003). 5181.

[13] C. Bhari, J.P. Draayer and S.A. Moszkowski, Phys. Rev. Lett. 68 (1992), 2133. 